University of Nebraska - Lincoln

DigitalCommons@University of Nebraska - Lincoln

\title{
A helical tow model and numerical simulation of on-line thermal curing of thermoset composites in filament winding
}

Xuefeng Wang

University of Nebraska-Lincoln

David Y.S. Lou

University of Nebraska-Lincoln, dlou1@unl.edu

Negli Zhang`

NASA Glenn Research Center, Lewis Field, Microgravity Science Division

Follow this and additional works at: https://digitalcommons.unl.edu/mechengfacpub

Part of the Mechanical Engineering Commons

Wang, Xuefeng; Lou, David Y.S.; and Zhang', Negli, "A helical tow model and numerical simulation of online thermal curing of thermoset composites in filament winding" (2004). Mechanical \& Materials Engineering Faculty Publications. 6.

https://digitalcommons.unl.edu/mechengfacpub/6

This Article is brought to you for free and open access by the Mechanical \& Materials Engineering, Department of at DigitalCommons@University of Nebraska - Lincoln. It has been accepted for inclusion in Mechanical \& Materials Engineering Faculty Publications by an authorized administrator of DigitalCommons@University of Nebraska Lincoln. 


\title{
A helical tow model and numerical simulation of on-line thermal curing of thermoset composites in filament winding
}

\author{
Xuefeng Wang a , David Y.S. Lou ${ }^{\text {a }}$, Nengli Zhang ${ }^{\mathrm{b}, *}$ \\ a Department of Mechanical Engineering, University of Nebraska, Lincoln, NE 68588, USA \\ ${ }^{\mathrm{b}}$ NASA Glenn Research Center, Lewis Field, Microgravity Science Division, 21000 Brookpark Road, Cleveland, OH 44135, USA
}

Received 10 March 2004; received in revised form 6 May 2004

Available online 22 July 2004

\begin{abstract}
A helical tow model of on-line curing of thermoset composites in winding is developed and solved numerically. Actual shape of tow in winding process is considered, and consequently, the modeling of the on-line curing process is more realistic. A numerical grid generation method is developed for the complicated geometry. Transformations of three-dimensional energy equation and its boundary conditions from physical domain to computational domain are performed, which are crucial in numerical grid generation method when an anisotropic medium is involved. A set of concise and regular equations is obtained. The numerical simulation results show that the helical tow model, in stead of a simplified model, should be used when the ratio of the diameter of fiber-wound composite structure to the diameter of tow is small.
\end{abstract}

(c) 2004 Elsevier Ltd. All rights reserved.

Keywords: Helical tow model; Curing; Filament winding; Numerical grid generation; Anisotropic medium

\section{Introduction}

Filament winding of thermoset composites are widely used in manufactures of high pressure vessels, tubings, and aerospace components. Traditional filament winding of thermoset composites is divided into two distinct processes: the winding of tow onto the mandrel at room temperature and the thermal processing of the whole composite structure in an autoclave or oven at high temperature so that the resin is cross linked via chemical reaction [1]. The thermal process, especially for large composite structures, in an autoclave or oven is timeconsuming. To overcome the disadvantages of the traditional manufacturing method of filament winding,

\footnotetext{
${ }^{*}$ Corresponding author. Tel.: +1-216-433-8750; fax: +1-216433-8050.

E-mail address: nengli.zhang@grc.nasa.gov (N. Zhang).
}

studies on on-line thermal processing of thermoset composites in filament winding have been conducted in recent years. Korotkov et al. [2] presented a onedimensional (radial direction) heat transfer model for the simultaneous processes of filament winding and curing of thermoset composites in which the surface of the composite was heated by infrared irradiation and a heated mandrel. Chern et al. [3] studied in situ curing for thermoset, hoop-wound structures using infrared heating. A two-dimensional (radial and azimuthal) model for infrared in situ curing was developed, in which the physical domain was mapped into a rectangular computational domain, neglecting the curvature of the surface layers. Recently, several models to simulate on-line curing of thermoset composites in filament winding have been developed by Wang and Lou [4-9]. A scheme of the on-site curing method of thermoset composite filament winding depicted by these models is shown in Fig. 1. In this method, the tow is locally heated by a radiative 


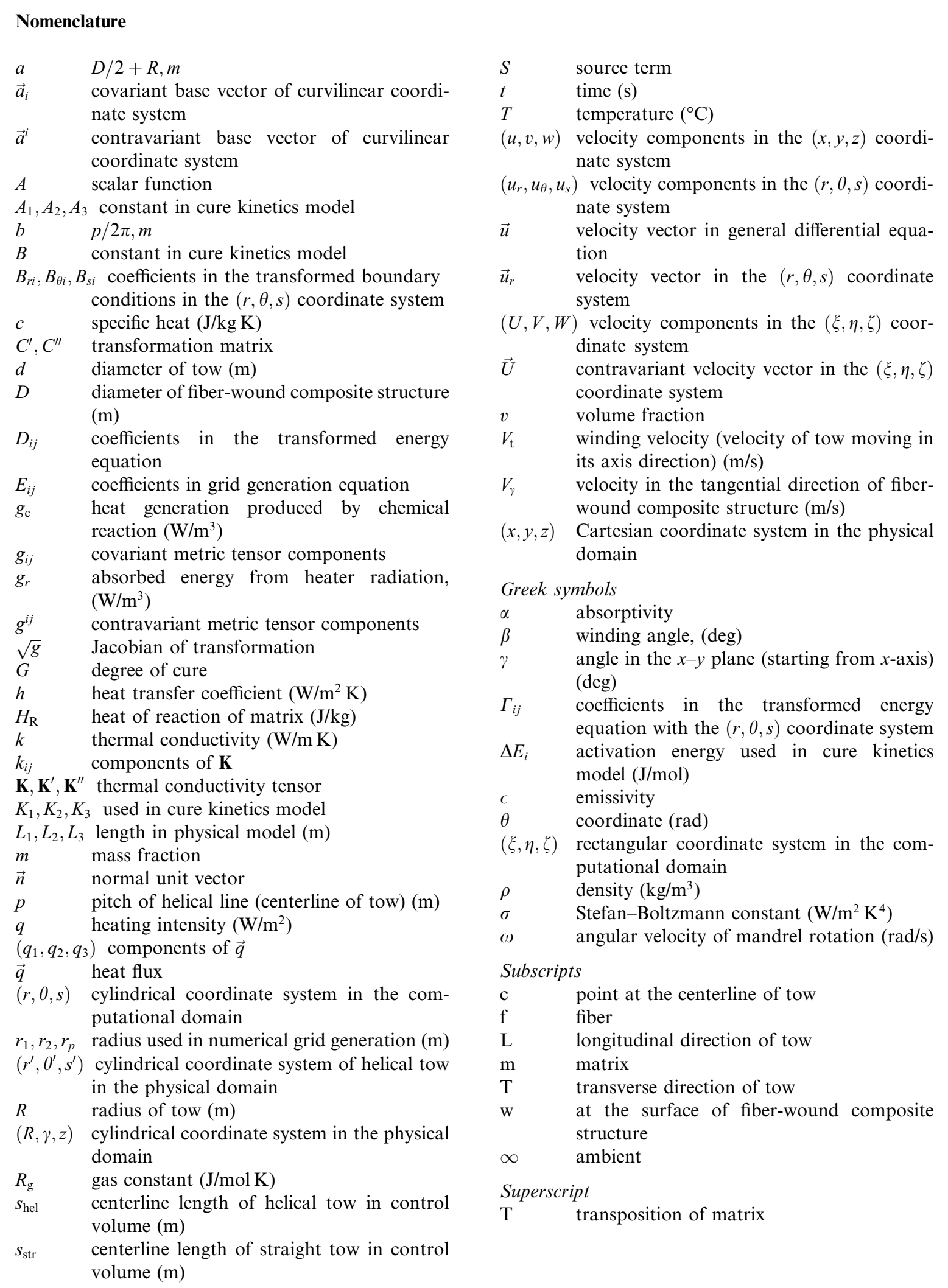




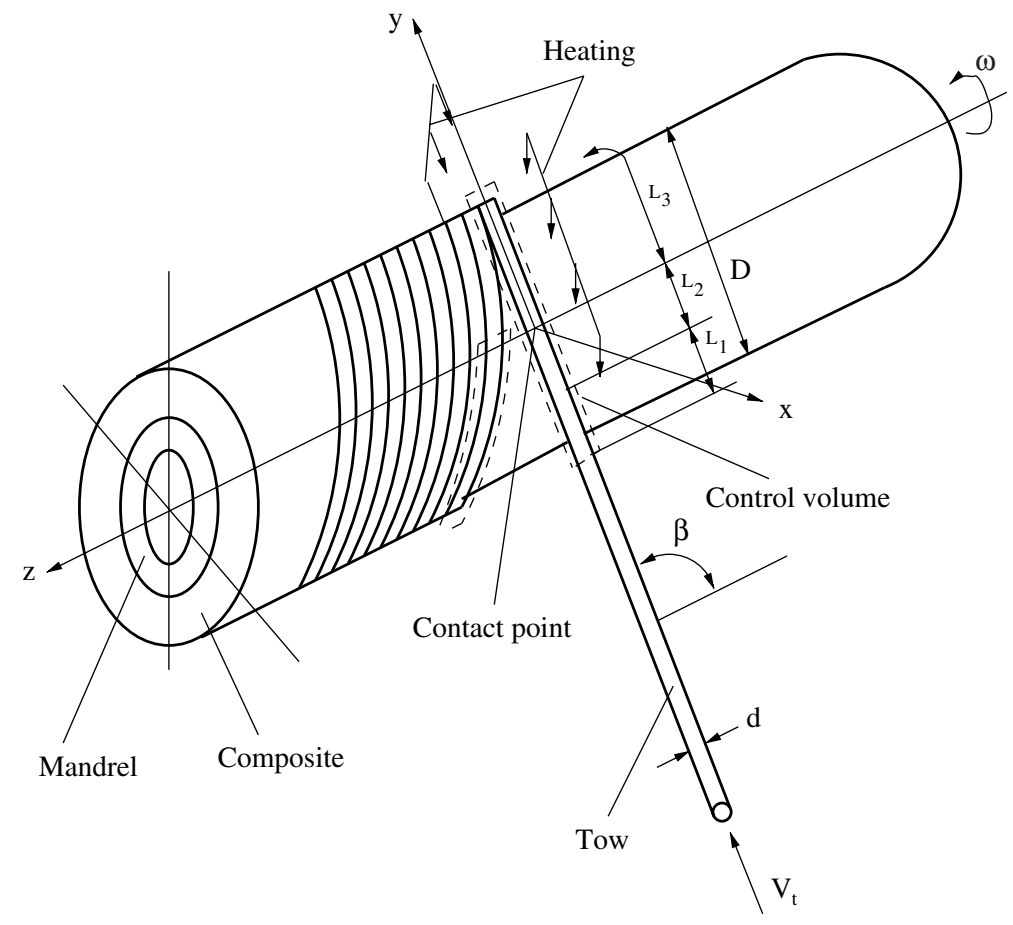

Fig. 1. Scheme of on-line thermal curing in filament winding.

heater, such as infrared, when it is wound onto the mandrel or the fiber-wound composite structure (hereafter simply referred as mandrel), so that the curing is done during the winding of the tow. The method offers many advantages over the traditional one, such as reduced residual stresses, easier production of composite structures with thick cross section, significant energy savings due to local heating, design flexibility, etc. [4]. In all of the previous models, tow is considered as a straight cylinder, neglecting the curvature of the mandrel. In fact, in the filament winding process, tow is wound helically as shown in Fig. 1. In this paper, the real shape of helical tow is considered in the on-line curing process and a numerical simulation of the three-dimensional process is performed.

During the past years, some studies on helical pipe flow problem have been conducted. Wang [10] introduced a non-orthogonal helical coordinate system along a spatial curve (the centerline of the helical pipe) in the study of the flow in a helical pipe. Later, by rotating the coordinate system introduced by Wang [10] around the centerline of helical pipe, Germano $[11,12]$ reported an orthogonal helical coordinate system along the centerline of helical pipe in his study on helical pipe flow. The governing equations in the helical coordinate system were given in Refs. [10-12]. Unfortunately, in the methods used by Wang [10] and Germano [11,12], governing equations (continuity equation, momentum equations, energy equation, etc.) in the helical coordi- nate system are too complicated to solve. On the other hand, the incoming tow before the contact point between the incoming tow and the mandrel in the filament winding process is straight instead of helical in shape, and therefore, the methods provided by Wang [10] and Germano [11] can not be used in the present study. Thompson et al. [13] introduced a general method of boundary-fitted coordinate system generation that can easily treat the fields with complex boundaries. The basic idea of the method is to make the boundaries to coincide with coordinate lines. These curvilinear coordinates coinciding with all boundaries are the solutions of an elliptic partial differential system in the physical domain with Dirichlet boundary conditions on all boundaries. One coordinate is specified to be constant on one of the boundaries, while a monotonic variation of the other coordinates around the boundary is specified. Therefore, each of the boundaries coincides with one of the coordinate lines. Using this numerical grid generation method, the problems with irregular geometries can be solved through the finite difference method or the finite volume method that has been widely used in numerical computations in heat transfer and fluid flow.

In the present study, the numerical grid generation method is successfully applied to the helical tow of on-line thermal curing of thermoset composites in filament winding. The transformed three-dimensional energy equation as well as boundary conditions with anisotropic thermal conductivities in the computational 
domain are derived and the transformed equation is numerically solved using finite volume method.

\section{Model analysis}

\subsection{Physical model}

Fig. 1 depicts the on-line thermal curing process in a Cartesian coordinate system $(x, y, z)$. The resin-impregnated tow, which is cylindrical shape with diameter $d$, is fed with a velocity $V_{\mathrm{t}}$ in the longitudinal direction of the tow onto the mandrel rotating at a speed $\omega$. The angle between incoming tow and the axis of the mandrel is defined as winding angle $\beta$. The strategy of on-line curing of thermoset composite in filament winding is to finish the curing of the tow during its winding process. To realize this strategy, heat source, such as infrared, is illuminated onto the region covering the portions of the tow just before and after the contact point between the incoming tow and the mandrel. A control volume that covers the interested portions of the tow is indicated by the dashed lines in Fig. 1 that is fixed relative to the heating source. The length of the tow within the control volume should be long enough to ensure that the curing has matured when the tow leaves the control volume. The temperature of the tow before entering the control volume is considered at environmental temperature $T_{\infty}$. The $z$-axis of the Cartesian coordinate system $(x, y, z)$ is placed along the centerline of mandrel and the $x$-axis goes through the contact point, as shown in Fig. 1. In this coordinate system, tow is spacially helical except the portion before the contact point. The physical domain of the helical tow model of the on-line curing is shown in Fig. 2. The tow is represented by a thick solid line in Fig. 2(a) for simplicity. The position of tow surface is depicted in Fig. 2(b), which is explained in detail in Section 3.2. The tow is considered to be made of fiber and resin that is uniformly distributed. The shape variation of the tow and the displacement of the resin in the radial direction of the tow during the filament winding process are very small that can be neglected in the thermal model [14]. The radiation energy from heat source is considered to be absorbed uniformly by the tow.

\subsection{Mathematical model}

For a practical on-line curing of thermoset composites in filament winding, a complex heat transfer process has to be considered. Several different heat transfer modes occur simultaneously, such as radiation from the heat source, heat conduction inside the tow and at the interface between the tow and the mandrel, and convective heat transfer between the surface of the tow and the surrounding atmosphere. The thermal conductivity of tow is orthotropic with respect to its principal axes.
Because of the motion of tow and the rotation of mandrel, a velocity field has to be also counted. Therefore, a three-dimensional energy equation for anisotropic medium is considered, which is

$$
\begin{aligned}
c[ & \left.\frac{\partial(\rho T)}{\partial t}+\frac{\partial}{\partial x}(\rho u T)+\frac{\partial}{\partial y}(\rho v T)+\frac{\partial}{\partial z}(\rho w T)\right] \\
= & \frac{\partial}{\partial x}\left(k_{11} \frac{\partial T}{\partial x}+k_{12} \frac{\partial T}{\partial y}+k_{13} \frac{\partial T}{\partial z}\right) \\
& +\frac{\partial}{\partial y}\left(k_{21} \frac{\partial T}{\partial x}+k_{22} \frac{\partial T}{\partial y}+k_{23} \frac{\partial T}{\partial z}\right) \\
& +\frac{\partial}{\partial z}\left(k_{31} \frac{\partial T}{\partial x}+k_{32} \frac{\partial T}{\partial y}+k_{33} \frac{\partial T}{\partial z}\right)+g_{c}+g_{r}
\end{aligned}
$$

where $g_{\mathrm{c}}$ is the heat generation from chemical reaction within resin, $g_{r}$ is the heat coming from radiation of heat source, $\rho$ is the density of the tow, $c$ is the specific heat of the tow, $k$ is the thermal conductivity of the tow, and $(u, v, w)$ are velocity components of the tow.

The boundary conditions of this model are very complicated. For the incoming tow before the contact point, the heater illuminates the portion $L_{2}$ where the surface of the tow undergoes radiation heat transfer and convection heat transfer with surroundings, while the portion $L_{1}$ undergoes convection heat transfer with surroundings only. For the tow after the contact point, the heater illuminates the portion $L_{3}$. The top surface of the tow is under the conditions of radiation and convection heat transfer with surroundings, while the under surface of the tow that contacts intimately with the surface of the mandrel is assumed as an isothermal boundary. The centerline of the tow is not at adiabatic due to the non-axisymmetric boundary condition on the tow surface. Boundary condition at the centerline of the tow needs to be specially treated. Here an approximation method - energy balance within an infinitesimally small unit around the centerline of the tow is considered [4]. At the front end of the control volume where the tow enters, the temperature of the tow equals to environmental temperature. The temperature at the other end of the control volume can be assumed to be fully developed with zero temperature-gradient in the axial direction of the tow because of the long enough domain.

\subsection{Cure kinetics model}

The heat generation due to chemical reaction within the resin during the curing process, $g_{\mathrm{c}}$, is given by

$g_{\mathrm{c}}=\rho_{\mathrm{m}} v_{\mathrm{m}}\left(\frac{\mathrm{d} G}{\mathrm{~d} t}\right) H_{\mathrm{R}}$

where $\rho_{\mathrm{m}}$ is the density of the matrix (i.e., resin) in the tow, $v_{\mathrm{m}}$ is the volume fraction of the matrix, $G$ is degree of cure, and $H_{\mathrm{R}}$ is the heat of reaction in the matrix. 

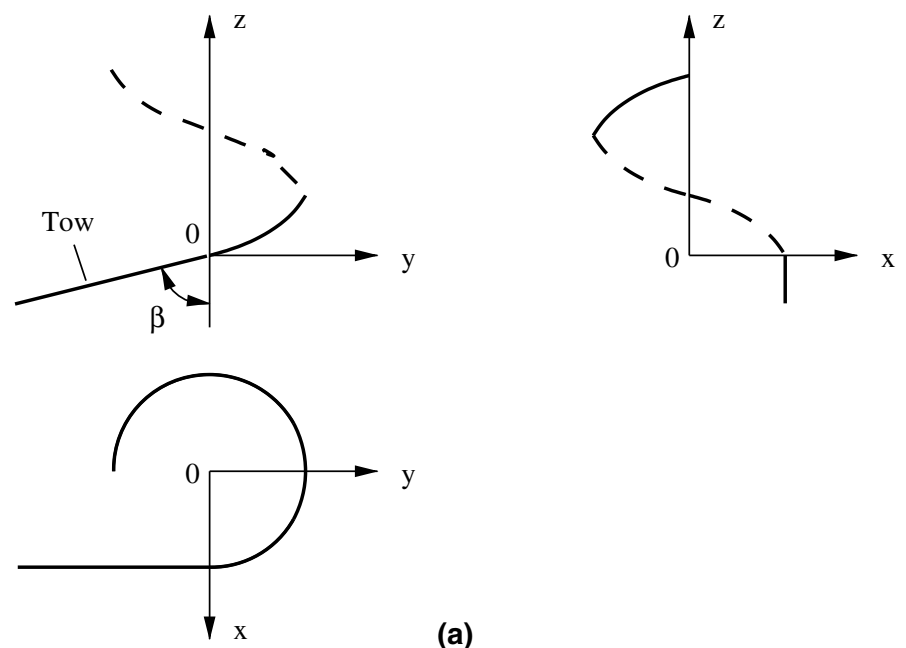

(a)

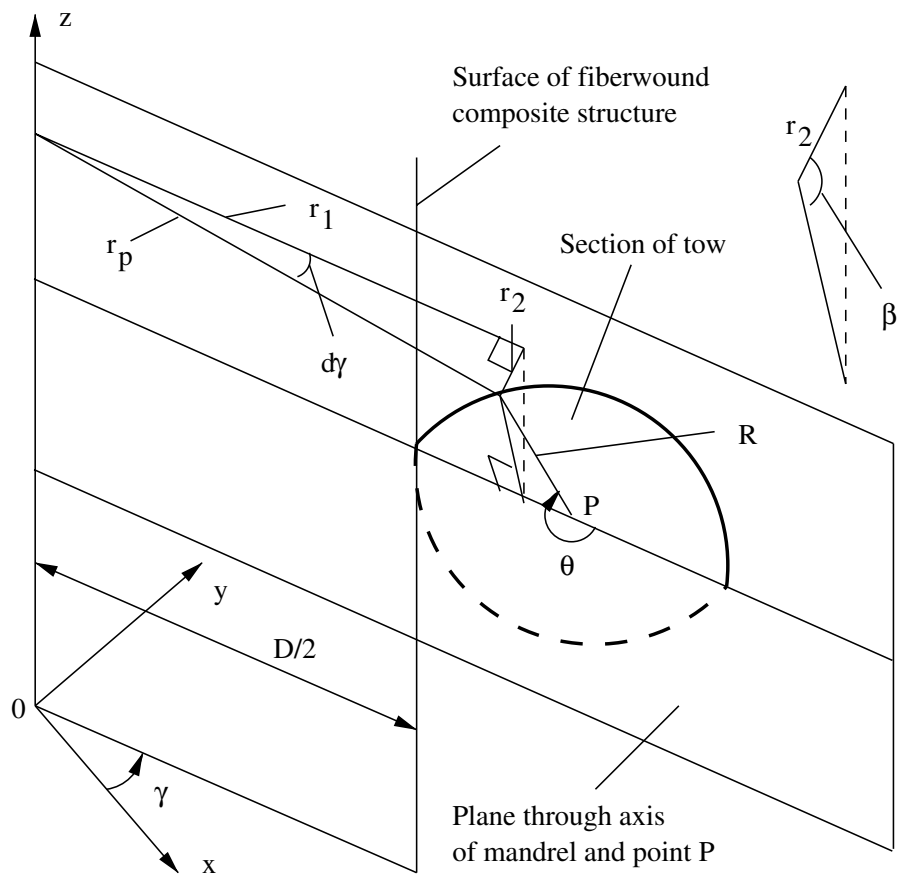

(b)

Fig. 2. Physical domain of helical tow model shown in the $(x, y, z)$ coordinate system: (a) projections on the different coordinate planes, (b) diagram to determine the position of tow surface.

In the studies of curing of thermoset resin, a cure kinetics model for Hercules 3501-6 resin developed by Lee et al. [15] has been widely used in engineering applications $[1,3]$. In this model, the heat release rate is defined as a function of cure degree and temperature. The cure degree is defined as the ratio of the exothermic heat release up to a given time to the heat of reaction that is the total heat release when all cross-linking reactions are completed.

Accordingly,

$$
\begin{aligned}
& \frac{\mathrm{d} G}{\mathrm{~d} t}=\left(K_{1}+K_{2} G\right)(1-G)(B-G) \quad \text { for } G \leqslant 0.3 \\
& \frac{\mathrm{d} G}{\mathrm{~d} t}=K_{3}(1-G) \quad \text { for } G>0.3
\end{aligned}
$$


with

$K_{1}=A_{1} \exp \left(-\Delta E_{1} / R_{\mathrm{g}} T\right), K_{2}=A_{2} \exp \left(-\Delta E_{2} / R_{\mathrm{g}} T\right)$,

$K_{3}=A_{3} \exp \left(-\Delta E_{3} / R_{\mathrm{g}} T\right)$

where $\left(B, A_{1}, A_{2}, A_{3}\right)$ are constants, $\Delta E$ is activation energy, and $R_{\mathrm{g}}$ is the gas constant. Applying the model to the present study, the values of the relevant parameters are given as: $H_{\mathrm{R}}=473.6 \pm 5.4 \mathrm{~J} / \mathrm{g}, B=0.47 \pm 0.07, A_{1}=$ $2.101 \times 10^{9} \mathrm{~min}^{-1}, A_{2}=-2.014 \times 10^{9} \mathrm{~min}^{-1}, A_{3}=1.960 \times$ $10^{5} \mathrm{~min}^{-1}, \Delta E_{1}=8.07 \times 10^{4} \mathrm{~J} / \mathrm{mol}, \Delta E_{2}=7.78 \times 10^{4} \mathrm{~J} /$ $\mathrm{mol}, \Delta E_{3}=5.66 \times 10^{4} \mathrm{~J} / \mathrm{mol}$.

It is assumed that the incoming tow is uncured, i.e., $G=0$. During the curing process, the cure degree of the resin increases gradually until it is completely cured.

\subsection{Material properties and relevant parameters}

The tow used in this study is Hercules AS/3501-6 prepreg in which fiber is carbon and resin is epoxy. The physical properties of fiber and resin can be found in Ref. [16]: resin density $\rho_{\mathrm{m}}=1.26 \times 10^{3} \mathrm{~kg} / \mathrm{m}^{3}$, fiber density $\rho_{\mathrm{f}}=1.79 \times 10^{3} \mathrm{~kg} / \mathrm{m}^{3}$, resin specific heat $c_{\mathrm{m}}=1260 \mathrm{~J} / \mathrm{kg} \mathrm{K}$, fiber specific heat $c_{\mathrm{f}}=712 \mathrm{~J} / \mathrm{kg} \mathrm{K}$, resin thermal conductivity $k_{\mathrm{m}}=0.167 \mathrm{~W} / \mathrm{m} \mathrm{K}$, fiber thermal conductivity $k_{\mathrm{f}}=26.0 \mathrm{~W} / \mathrm{m} \mathrm{K}$. For the resin impregnated fiber tow, properties are given for the combination of those of both fiber and resin. Because tow is made of unidirectional fiber and resin, its thermal conductivity is orthotropic and the principal directions are parallel and normal to the fiber, respectively. According to Refs. [17,18], the relevant properties of the tow are

the density $\rho=\rho_{\mathrm{m}} v_{\mathrm{m}}+\rho_{\mathrm{f}} v_{\mathrm{f}}$

the specific heat $c=c_{\mathrm{m}} m_{\mathrm{m}}+c_{\mathrm{f}} m_{\mathrm{f}}$

the thermal conductivities $k_{\mathrm{L}}=k_{\mathrm{m}} v_{\mathrm{m}}+k_{\mathrm{f}} v_{\mathrm{f}}$

$k_{\mathrm{T}}=\frac{k_{\mathrm{m}} k_{\mathrm{f}}}{k_{\mathrm{m}} v_{\mathrm{f}}+k_{\mathrm{f}} v_{\mathrm{m}}}$

where subscripts $\mathrm{L}$ and $\mathrm{T}$ refer to thermal conductivities in the directions parallel $\left(k_{\mathrm{L}}\right)$ and normal $\left(k_{\mathrm{T}}\right)$ to the filament, respectively. The mass fraction of resin, defined as the percentage of the mass of resin to the mass of tow, and the mass fraction of fiber that is the percentage of the mass of fiber to the mass of tow, can be obtained by calculations using the following relations:

$m_{\mathrm{f}}=\frac{\rho_{\mathrm{f}} v_{\mathrm{f}}}{\rho}, \quad m_{\mathrm{m}}=\frac{\rho_{\mathrm{m}} v_{\mathrm{m}}}{\rho}$

In the present study, absorptivity of the tow is conservatively assumed to be 0.8 , which is generally over $80 \%$ $[19,20]$. According to existing practice, the relevant parameters used in the calculation are: diameter of the mandrel $D=0.2 \mathrm{~m}$, diameter of the tow $d=0.002 \mathrm{~m}$, volume fraction of resin $v_{\mathrm{m}}=0.2$, volume fraction of fiber $v_{\mathrm{f}}=0.8$, absorptivity $\alpha=0.8$, emissivity $\epsilon=0.8$, Stefan-Boltzmann constant $\sigma=5.675 \times 10^{-8} \mathrm{~W} / \mathrm{m}^{2} \mathrm{~K}^{4}$. Because the mandrel is always rotated during the filament winding process, the convective heat transfer coefficient on the surface of the tow is evaluated using the empirical correlation for an isothermal, rotating cylinder in air [4,21].

\section{Numerical grid generation}

The geometry of the physical domain of the abovementioned physical model is too complicated to handle directly by the finite difference method or the finite volume method. In order to apply the finite volume method to the present study, the numerical grid generation method is used to transform the physical domain of irregular geometry into a computational domain of regular geometry. All calculations can be carried out in the computational domain. Details of the transformation and the numerical calculation method can be found in Ref. [4].

\subsection{Partial differential equations of grid generation}

Generally, a computational domain of simple regular geometry should be chosen in the numerical grid generation method. Accordingly, the tow of the helical cylinder in filament winding is considered as a straight cylinder in the computational domain. Correspondingly, a rectangular coordinate system $(\xi, \eta, \zeta)$ is established first. Coordinates $\xi$ and $\eta$ are in the cross section of the straight cylinder, and $\zeta$ is along the axial direction of the straight cylinder. Because a cylindrical coordinate system is preferred in numerical calculations for the cylinder, the cylindrical coordinate system $(r, \theta, s)$ is chosen as the final coordinate system in the computational domain, where $r$ is in the radial direction, $\theta$ is in the azimuthal direction, and $s$ follows the axial direction of the straight cylinder. The actual coordinate transformation is from $(x, y, z)$ to $(\xi, \eta, \zeta)$, and then from $(\xi, \eta, \zeta)$ to $(r, \theta, s)$. The transformation from the Cartesian coordinates $(x, y, z)$ in the physical domain to the Cartesian coordinates $(\xi, \eta, \zeta)$ in the computational domain is performed using the numerical grid generation method. In the computational domain, the transformation from the Cartesian coordinates $(\xi, \eta, \zeta)$ to the cylindrical coordinates $(r, \theta, s)$ can be realized through an algebraic transformation. The basic algebraic relationships between the two coordinate systems $(\xi, \eta, \zeta)$ and $(r, \theta, s)$ are

$\xi=r \cos \theta, \quad \eta=r \sin \theta, \quad \zeta=s$

Being the function in $r, \theta$, and $s$, the covariant base vectors are 


$$
\begin{aligned}
\vec{a}_{1}= & x_{\xi} \vec{i}+y_{\xi} \vec{j}+z_{\xi} \vec{k} \\
= & {\left[\left(x_{r} \cos \theta-x_{\theta} \frac{\sin \theta}{r}\right) \cos \theta+\left(y_{r} \cos \theta\right.\right.} \\
& \left.\left.-y_{\theta} \frac{\sin \theta}{r}\right) \sin \theta\right] \vec{e}_{r}+\left[\left(-x_{r} \cos \theta+x_{\theta} \frac{\sin \theta}{r}\right) \sin \theta\right. \\
& \left.+\left(y_{r} \cos \theta-y_{\theta} \frac{\sin \theta}{r}\right) \cos \theta\right] \vec{e}_{\theta} \\
& +\left[z_{r} \cos \theta-z_{\theta} \frac{\sin \theta}{r}\right] \vec{e}_{s} \\
\vec{a}_{2}= & x_{\eta} \vec{i}+y_{\eta} \vec{j}+z_{\eta} \vec{k} \\
= & {\left[\left(x_{r} \sin \theta+x_{\theta} \frac{\cos \theta}{r}\right) \cos \theta+\left(y_{r} \sin \theta\right.\right.} \\
& \left.\left.+y_{\theta} \frac{\cos \theta}{r}\right) \sin \theta\right] \vec{e}_{r}+\left[\left(-x_{r} \sin \theta-x_{\theta} \frac{\cos \theta}{r}\right) \sin \theta\right. \\
& \left.+\left(y_{r} \sin \theta+y_{\theta} \frac{\cos \theta}{r}\right) \cos \theta\right] \vec{e}_{\theta} \\
& +\left[z_{r} \sin \theta+z_{\theta} \frac{\cos \theta}{r}\right] \vec{e}_{s} \\
\vec{a}_{3}= & x_{\zeta} \vec{i}+y_{\zeta} \vec{j}+z_{\zeta} \vec{k} \\
= & \left.x_{s} \cos \theta+y_{s} \sin \theta\right] \vec{e}_{r}+\left[-x_{s} \sin \theta+y_{s} \cos \theta\right] \vec{e}_{\theta}+\left[z_{s}\right] \vec{e}_{s} \\
& {[9) } \\
&
\end{aligned}
$$

To overcome the singularity at $r=0$, the L'Hospital's rule is used. The covariant metric tensor is

$g_{i j}=\vec{a}_{i} \cdot \vec{a}_{j}=g_{j i} \quad(i=1,2,3), \quad(j=1,2,3)$

and the Jacobian of the transformation is

$\sqrt{g}=\sqrt{\operatorname{det}\left|g_{i j}\right|}=\vec{a}_{1} \cdot\left(\vec{a}_{2} \times \vec{a}_{3}\right)$

The contravariant base vectors are

$\vec{a}^{i}=\nabla \xi^{i}=\frac{1}{\sqrt{g}} \vec{a}_{j} \times \vec{a}_{k} \quad(i=1,2,3), \quad(i, j, k)$ cyclic

The contravariant metric tensor is

$g^{i j}=\vec{a}^{i} \cdot \vec{a}^{j}=g^{j i} \quad(i=1,2,3), \quad(j=1,2,3)$

For numerical grid generation, the coordinates are solutions of the chosen partial differential equations. As well known, elliptic type differential equations are generally used because of their smoothing effect in spreading out the boundary slope irregularities [22]. On the other hand, the Laplace system is preferred for its simplicity. Therefore, elliptic grid generation method with Laplace system is adopted in the present study. The Laplace grid generation equations to be solved are

$\nabla^{2} A=0$

where $A$ represents $x, y$, and $z$. The expression for $\nabla^{2} A$ is given by

$$
\begin{aligned}
\sqrt{g} \nabla^{2} A= & \frac{\partial}{\partial \xi}\left(\sqrt{g} g^{11} \frac{\partial A}{\partial \xi}\right)+\frac{\partial}{\partial \xi}\left(\sqrt{g} g^{12} \frac{\partial A}{\partial \eta}\right) \\
& +\frac{\partial}{\partial \xi}\left(\sqrt{g} g^{13} \frac{\partial A}{\partial \zeta}\right)+\frac{\partial}{\partial \eta}\left(\sqrt{g} g^{21} \frac{\partial A}{\partial \xi}\right) \\
& +\frac{\partial}{\partial \eta}\left(\sqrt{g} g^{22} \frac{\partial A}{\partial \eta}\right)+\frac{\partial}{\partial \eta}\left(\sqrt{g} g^{23} \frac{\partial A}{\partial \zeta}\right) \\
& +\frac{\partial}{\partial \zeta}\left(\sqrt{g} g^{31} \frac{\partial A}{\partial \xi}\right)+\frac{\partial}{\partial \zeta}\left(\sqrt{g} g^{32} \frac{\partial A}{\partial \eta}\right) \\
& +\frac{\partial}{\partial \zeta}\left(\sqrt{g} g^{33} \frac{\partial A}{\partial \zeta}\right)
\end{aligned}
$$

In the cylindrical coordinate system $(r, \theta, s)$, it becomes

$$
\begin{aligned}
\sqrt{g} \nabla^{2} A= & \frac{1}{r} \frac{\partial}{\partial r}\left[r E_{11} \frac{\partial A}{\partial r}\right]+\frac{1}{r} \frac{\partial}{\partial r}\left[r E_{12} \frac{1}{r} \frac{\partial A}{\partial \theta}\right] \\
& +\frac{1}{r} \frac{\partial}{\partial r}\left[r E_{13} \frac{\partial A}{\partial s}\right]+\frac{1}{r} \frac{\partial}{\partial \theta}\left[E_{21} \frac{\partial A}{\partial r}\right] \\
& +\frac{1}{r} \frac{\partial}{\partial \theta}\left[E_{22} \frac{1}{r} \frac{\partial A}{\partial \theta}\right]+\frac{1}{r} \frac{\partial}{\partial \theta}\left[E_{23} \frac{\partial A}{\partial s}\right] \\
& +\frac{\partial}{\partial s}\left[E_{31} \frac{\partial A}{\partial r}\right]+\frac{\partial}{\partial s}\left[E_{32} \frac{1}{r} \frac{\partial A}{\partial \theta}\right]+\frac{\partial}{\partial s}\left[E_{33} \frac{\partial A}{\partial s}\right]
\end{aligned}
$$

where

$$
\begin{aligned}
E_{11}= & \sqrt{g} g^{11} \cos ^{2} \theta+\sqrt{g} g^{12} \sin 2 \theta+\sqrt{g} g^{22} \sin ^{2} \theta \\
E_{12}= & E_{21}=-\sqrt{g} g^{11} \sin \theta \cos \theta \\
& +\sqrt{g} g^{12} \cos 2 \theta+\sqrt{g} g^{22} \sin \theta \cos \theta \\
E_{13}= & E_{31}=\sqrt{g} g^{13} \cos \theta+\sqrt{g} g^{23} \sin \theta \\
E_{22}= & \sqrt{g} g^{11} \sin ^{2} \theta-\sqrt{g} g^{12} \sin 2 \theta+\sqrt{g} g^{22} \cos ^{2} \theta \\
E_{23}= & E_{32}=-\sqrt{g} g^{13} \sin \theta+\sqrt{g} g^{23} \cos \theta \\
E_{33}= & \sqrt{g} g^{33}
\end{aligned}
$$

\subsection{Boundary conditions for grid generation equations}

The coordinate values of points on the boundary of each cross section of the helical tow can be obtained based on the coordinate value of its center point $P$, as shown in Fig. 2(b). Let $a$ be the radius of point $P, \gamma$ be the angle of point $P$ in $x-y$ plane starting from $x$-axis, and $p$ be the pitch of helical line, then

$$
\begin{aligned}
& a=\frac{D}{2}+R, \quad p=2 \pi a \cot \beta, \quad b=\frac{p}{2 \pi}, \\
& \gamma=\frac{s_{\text {hel }}}{\sqrt{a^{2}+b^{2}}}
\end{aligned}
$$

where $s_{\text {hel }}$ is the centerline length of the helical tow in the control volume. The relevant parameters shown in Fig. 2(b) are

$$
\begin{aligned}
& r_{1}=a+R \cos \theta, \quad r_{2}=R \sin \theta \cos \beta, \\
& r_{p}=\sqrt{r_{1}^{2}+r_{2}^{2}}, \quad \tan (\mathrm{d} \gamma)=\frac{r_{2}}{r_{1}}
\end{aligned}
$$


The coordinate values of point $P$ at the centerline of the helical tow are

$$
\begin{aligned}
& x_{\mathrm{c}}=a \cos \frac{s_{\mathrm{hel}}}{\sqrt{a^{2}+b^{2}}}, \quad y_{\mathrm{c}}=a \sin \frac{s_{\mathrm{hel}}}{\sqrt{a^{2}+b^{2}}}, \\
& z_{\mathrm{c}}=\frac{b s_{\text {hel }}}{\sqrt{a^{2}+b^{2}}}
\end{aligned}
$$

For the straight tow before the contact point, the coordinate values of point $P$ can be easily determined as

$x_{\mathrm{c}}=a, \quad y_{\mathrm{c}}=s_{\mathrm{str}} \sin \beta, \quad z_{\mathrm{c}}=s_{\mathrm{str}} \cos \beta$

The coordinate values of points on the surface of the helical tow are

$$
\begin{aligned}
& x=r_{p} \cos (\gamma+\mathrm{d} \gamma), \quad y=r_{p} \sin (\gamma+\mathrm{d} \gamma), \\
& z=z_{\mathrm{c}}-R \sin \theta \sin \beta
\end{aligned}
$$

while the surface of the straight tow before the contact point is described by

$x=x_{\mathrm{c}}+R \cos \theta, \quad y=y_{\mathrm{c}}+R \sin \theta \cos \beta$,

$z=z_{\mathrm{c}}-R \sin \theta \sin \beta$

\section{Solution of grid generation equations and transforma- tion of energy equation}

The grid generation equations, Eq. (15), are solved numerically using a successive over- relaxation (SOR) iterative method. For the derivatives of $x, y, z$ with respect to the coordinates $r, \theta, s$ the central difference scheme is used for the middle points and the second order forward or backward three-point difference scheme are used for the boundary points.

The transformation of governing equations from the physical domain to the computational domain is essential to the numerical grid generation method. The energy equation in tensor form is given by

$c\left[\frac{\partial(\rho T)}{\partial t}+\nabla \cdot(\rho T \vec{u})\right]=-\nabla \cdot \vec{q}+S$

or, in the Cartesian coordinate system $(x, y, z)$

$$
\begin{gathered}
c\left[\frac{\partial(\rho T)}{\partial t}+\frac{\partial}{\partial x}(\rho u T)+\frac{\partial}{\partial y}(\rho v T)+\frac{\partial}{\partial z}(\rho w T)\right] \\
=\frac{\partial}{\partial x}\left(k_{11} \frac{\partial T}{\partial x}+k_{12} \frac{\partial T}{\partial y}+k_{13} \frac{\partial T}{\partial z}\right) \\
\quad+\frac{\partial}{\partial y}\left(k_{21} \frac{\partial T}{\partial x}+k_{22} \frac{\partial T}{\partial y}+k_{23} \frac{\partial T}{\partial z}\right) \\
+\frac{\partial}{\partial z}\left(k_{31} \frac{\partial T}{\partial x}+k_{32} \frac{\partial T}{\partial y}+k_{33} \frac{\partial T}{\partial z}\right)+S
\end{gathered}
$$

where source term $S$ represents all heat generations. After the energy equation is transformed to the com- putational domain in $(\xi, \eta, \zeta)$ coordinate system, it becomes

$$
\begin{gathered}
c\left[\frac{\partial(\rho T)}{\partial t}+\frac{1}{\sqrt{g}} \sum_{i=1}^{3}\left(\sqrt{g} \rho T \vec{a}^{i} \cdot \vec{u}\right)_{\xi^{i}}\right] \\
=-\frac{1}{\sqrt{g}} \sum_{i=1}^{3}\left(\sqrt{g} \vec{a}^{i} \cdot \vec{q}\right)_{\xi^{i}}+S
\end{gathered}
$$

where $\xi^{i}$ represents $\xi, \eta, \zeta$ when $i=1,2,3$, respectively.

For anisotropic medium, the heat flux is

$\vec{q}=q_{1} \vec{i}+q_{2} \vec{j}+q_{3} \vec{k}$

where

$q_{i}=-\sum_{j=1}^{3} k_{i j} \frac{\partial T}{\partial x_{j}}$

After transformation

$q_{i}=-\sum_{j=1}^{3} k_{i j}\left[\sum_{k=1}^{3} T_{\xi^{k}}\left(\xi^{k}\right) x_{j}\right]$

Then, the energy equation in the computational domain using the coordinate system $(\xi, \eta, \zeta)$ becomes

$$
\begin{aligned}
c[ & \left.\frac{\partial(\sqrt{g} \rho T)}{\partial T}+\frac{\partial}{\partial \xi}(\sqrt{g} \rho U T)+\frac{\partial}{\partial \eta}(\sqrt{g} \rho V T)+\frac{\partial}{\partial \zeta}(\sqrt{g} \rho W T)\right] \\
= & \frac{\partial}{\partial \xi}\left(\sqrt{g} D_{11} \frac{\partial T}{\partial \xi}\right)+\frac{\partial}{\partial \xi}\left(\sqrt{g} D_{12} \frac{\partial T}{\partial \eta}\right) \\
& +\frac{\partial}{\partial \xi}\left(\sqrt{g} D_{13} \frac{\partial T}{\partial \zeta}\right)+\frac{\partial}{\partial \eta}\left(\sqrt{g} D_{21} \frac{\partial T}{\partial \xi}\right) \\
& +\frac{\partial}{\partial \eta}\left(\sqrt{g} D_{22} \frac{\partial T}{\partial \eta}\right)+\frac{\partial}{\partial \eta}\left(\sqrt{g} D_{23} \frac{\partial T}{\partial \zeta}\right) \\
& +\frac{\partial}{\partial \zeta}\left(\sqrt{g} D_{31} \frac{\partial T}{\partial \xi}\right)+\frac{\partial}{\partial \zeta}\left(\sqrt{g} D_{32} \frac{\partial T}{\partial \eta}\right) \\
& +\frac{\partial}{\partial \zeta}\left(\sqrt{g} D_{33} \frac{\partial T}{\partial \zeta}\right)+\sqrt{g} S
\end{aligned}
$$

where

$$
\begin{aligned}
D_{11}= & k_{11} \xi_{x} \xi_{x}+k_{12} \xi_{y} \xi_{x}+k_{13} \xi_{z} \xi_{x}+k_{21} \xi_{x} \xi_{y} \\
& +k_{22} \xi_{y} \xi_{y}+k_{23} \xi_{z} \xi_{y}+k_{31} \xi_{x} \xi_{z}+k_{32} \xi_{y} \xi_{z} \\
& +k_{33} \xi_{z} \xi_{z} \\
D_{12}= & D_{21} \\
= & k_{11} \eta_{x} \xi_{x}+k_{12} \eta_{y} \xi_{x}+k_{13} \eta_{z} \xi_{x}+k_{21} \eta_{x} \xi_{y} \\
& +k_{22} \eta_{y} \xi_{y}+k_{23} \eta_{z} \xi_{y}+k_{31} \eta_{x} \xi_{z}+k_{32} \eta_{y} \xi_{z} \\
& +k_{33} \eta_{z} \xi_{z}
\end{aligned}
$$




$$
\begin{aligned}
D_{13}= & D_{31} \\
= & k_{11} \zeta_{x} \xi_{x}+k_{12} \zeta_{y} \xi_{x}+k_{13} \zeta_{z} \xi_{x}+k_{21} \zeta_{x} \xi_{y} \\
& +k_{22} \zeta_{y} \xi_{y}+k_{23} \zeta_{z} \xi_{y}+k_{31} \zeta_{x} \xi_{z}+k_{32} \zeta_{y} \xi_{z} \\
& +k_{33} \zeta_{z} \xi_{z} \\
D_{22}= & k_{11} \eta_{x} \eta_{x}+k_{12} \eta_{y} \eta_{x}+k_{13} \eta_{z} \eta_{x}+k_{21} \eta_{x} \eta_{y} \\
& +k_{22} \eta_{y} \eta_{y}+k_{23} \eta_{z} \eta_{y}+k_{31} \eta_{x} \eta_{z}+k_{32} \eta_{y} \eta_{z} \\
& +k_{33} \eta_{z} \eta_{z} \\
D_{23}= & D_{32} \\
= & k_{11} \zeta_{x} \eta_{x}+k_{12} \zeta_{y} \eta_{x}+k_{13} \zeta_{z} \eta_{x}+k_{21} \zeta_{x} \eta_{y} \\
& +k_{22} \zeta_{y} \eta_{y}+k_{23} \zeta_{z} \eta_{y}+k_{31} \zeta_{x} \eta_{z}+k_{32} \zeta_{y} \eta_{z} \\
& +k_{33} \zeta_{z} \eta_{z} \\
= & k_{11} \zeta_{x} \zeta_{x}+k_{12} \zeta_{y} \zeta_{x}+k_{13} \zeta_{z} \zeta_{x}+k_{21} \zeta_{x} \zeta_{y}+k_{22} \zeta_{y} \zeta_{y} \\
& +k_{23} \zeta_{z} \zeta_{y}+k_{31} \zeta_{x} \zeta_{z}+k_{32} \zeta_{y} \zeta_{z}+k_{33} \zeta_{z} \zeta_{z}
\end{aligned}
$$

As mentioned above, the governing equations (momentum equations, energy equation, etc.) are very complicated in the method introduced by Wang [10] and Germano $[11,12]$. There are too many additional differential terms in the governing equations to handle. Additionally, when applying the numerical grid generation method to an anisotropic medium, the transformed two-dimensional energy equation is very complex and contains too many differential terms to be solved [22-24]. It also should be noted that most studies on the fluid flow problems have not considered any anisotropic property. Contrastedly, Eq. (32) is threedimensional with a tidy format for an anisotropic medium, and very suitable for the finite volume method. Obviously, the transformed three-dimensional energy equation for an anisotropic medium developed in the present study is very significant and meaningful.

Finally, the transformed energy equation in the cylindrical coordinate system $(r, \theta, s)$ is given by

$$
\begin{gathered}
c\left[\frac{\partial(\sqrt{g} \rho T)}{\partial t}+\frac{1}{r} \frac{\partial}{\partial r}\left(r \sqrt{g} \rho u_{r} T\right)+\frac{1}{r} \frac{\partial}{\partial \theta}\left(\sqrt{g} \rho u_{\theta} T\right)\right. \\
\left.+\frac{\partial}{\partial s}\left(\sqrt{g} \rho u_{s} T\right)\right] \\
=\frac{1}{r} \frac{\partial}{\partial r}\left(r \sqrt{g} \Gamma_{11} \frac{\partial T}{\partial r}\right)+\frac{1}{r} \frac{\partial}{\partial r}\left(r \sqrt{g} \Gamma_{12} \frac{1}{r} \frac{\partial T}{\partial \theta}\right) \\
+\frac{1}{r} \frac{\partial}{\partial r}\left(r \sqrt{g} \Gamma_{13} \frac{\partial T}{\partial s}\right)+\frac{1}{r} \frac{\partial}{\partial \theta}\left(\sqrt{g} \Gamma_{21} \frac{\partial T}{\partial r}\right) \\
+\frac{1}{r} \frac{\partial}{\partial \theta}\left(\sqrt{g} \Gamma_{22} \frac{1}{r} \frac{\partial T}{\partial \theta}\right)+\frac{1}{r} \frac{\partial}{\partial \theta}\left(\sqrt{g} \Gamma_{23} \frac{\partial T}{\partial s}\right) \\
+\frac{\partial}{\partial s}\left(\sqrt{g} \Gamma_{31} \frac{\partial T}{\partial r}\right)+\frac{\partial}{\partial s}\left(\sqrt{g} \Gamma_{32} \frac{1}{r} \frac{\partial T}{\partial \theta}\right) \\
+\frac{\partial}{\partial s}\left(\sqrt{g} \Gamma_{33} \frac{\partial T}{\partial s}\right)+\sqrt{g} S
\end{gathered}
$$

where

$$
\begin{aligned}
& \Gamma_{11}=D_{11} \cos ^{2} \theta+D_{12} \sin 2 \theta+D_{22} \sin ^{2} \theta \\
& \Gamma_{12}=\Gamma_{21}=-D_{11} \sin \theta \cos \theta+D_{12} \cos 2 \theta+D_{22} \sin \theta \cos \theta
\end{aligned}
$$

$\Gamma_{13}=\Gamma_{31}=D_{13} \cos \theta+D_{23} \sin \theta$

$\Gamma_{22}=D_{11} \sin ^{2} \theta-D_{12} \sin 2 \theta+D_{22} \cos ^{2} \theta$

$\Gamma_{23}=\Gamma_{32}=-D_{13} \sin \theta+D_{23} \cos \theta$

$\Gamma_{33}=D_{33}$

The velocity field in the coordinate system $(r, \theta, s)$ is

$\vec{u}_{r}=u_{r} \vec{e}_{r}+u_{\theta} \vec{e}_{\theta}+u_{s} \vec{e}_{s}$

where

$$
\begin{aligned}
& u_{r}=U \cos \theta+V \sin \theta, \\
& u_{\theta}=-U \sin \theta+V \cos \theta, \quad u_{s}=W
\end{aligned}
$$

\subsection{Determination of velocity field}

In energy equation Eq. (27), convection terms account for the motion of the tow in filament winding. If the moving speed of tow in its axial direction is given as $V_{\mathrm{t}}$, then the angular velocity of mandrel rotation is

$\omega=\frac{V_{\mathrm{t}} \sin \beta}{a}$

Subsequently, the velocity in the tangential direction of the fiber-wound composite structure is

$V_{\gamma}=\sqrt{x^{2}+y^{2}} \omega$

If the velocity vector of the tow in the $(x, y, z)$ coordinate system is

$\vec{u}=u \vec{i}+v \vec{j}+w \vec{k}$

the velocity components of the helical tow are

$u=-V_{\gamma} \sin \gamma, \quad v=V_{\gamma} \cos \gamma, \quad w=V_{\mathrm{t}} \cos \beta$

The velocity components of the straight tow are

$u=0, \quad v=V_{\mathrm{t}} \sin \beta, \quad w=V_{\mathrm{t}} \cos \beta$

The contravariant velocity field in the $(\xi, \eta, \zeta)$ coordinate system is

$\vec{U}=U \vec{i}+V \vec{j}+W \vec{k}$

where

$U^{i}=\vec{a}^{i} \cdot \vec{u} \quad(i=1,2,3)$ 
or

$$
\begin{aligned}
& U=\frac{1}{\sqrt{g}}\left[u\left(y_{\eta} z_{\zeta}-z_{\eta} y_{\zeta}\right)+v\left(z_{\eta} x_{\zeta}-x_{\eta} z_{\zeta}\right)+w\left(x_{\eta} y_{\zeta}-y_{\eta} x_{\zeta}\right)\right] \\
& V=\frac{1}{\sqrt{g}}\left[u\left(y_{\zeta} z_{\xi}-z_{\zeta} y_{\xi}\right)+v\left(z_{\zeta} x_{\xi}-x_{\zeta} z_{\xi}\right)+w\left(x_{\zeta} y_{\xi}-y_{\zeta} x_{\xi}\right)\right] \\
& W=\frac{1}{\sqrt{g}}\left[u\left(y_{\xi} z_{\eta}-z_{\xi} y_{\eta}\right)+v\left(z_{\xi} x_{\eta}-x_{\xi} z_{\eta}\right)+w\left(x_{\xi} y_{\eta}-y_{\xi} x_{\eta}\right)\right]
\end{aligned}
$$

\subsection{Determination of thermal conductivity matrix}

The thermal conductivity tensor of helical tow in the cylindrical coordinate system $\left(r^{\prime}, \theta^{\prime}, s^{\prime}\right)$ is

$$
\left[\mathbf{K}^{\prime}\right]=\left[\begin{array}{ccc}
k_{\mathrm{T}} & 0 & 0 \\
0 & k_{\mathrm{T}} & 0 \\
0 & 0 & k_{\mathrm{L}}
\end{array}\right]
$$

In order to obtain the thermal conductivity tensor in the $(x, y, z)$ coordinate system, a transformation of thermal conductivity tensor $\mathbf{K}^{\prime}$ from the $\left(r^{\prime}, \theta^{\prime}, s^{\prime}\right)$ coordinate system into the cylindrical coordinate system $(R, \gamma, z)$, symbolized as $\mathbf{K}^{\prime \prime}$, is conducted first. These two coordinate systems are displayed in Fig. 3. In the coordinate system $\left(r^{\prime}, \theta^{\prime}, s^{\prime}\right), r^{\prime}$ is the radial direction of the tow, $\theta^{\prime}$ is the angle in the azimuthal direction of the tow, and $s^{\prime}$ is the axis of the tow. In the coordinate system $(R, \gamma, z), R$ is the radial direction of the mandrel, $\gamma$ is the angle in the azimuthal direction, and $z$ is the axis of the mandrel. The tensor $\mathbf{K}^{\prime \prime}$ is given by

$$
\begin{aligned}
{\left[\mathbf{K}^{\prime \prime}\right] } & =\left[C^{\prime}\right]\left[\mathbf{K}^{\prime}\right]\left[C^{\prime}\right]^{\mathrm{T}} \\
& =\left[\begin{array}{ccc}
k_{\mathrm{T}} & 0 & 0 \\
0 & k_{\mathrm{T}} \cos ^{2} \beta+k_{\mathrm{L}} \sin ^{2} \beta & \left(k_{\mathrm{L}}-k_{\mathrm{T}}\right) \sin \beta \cos \beta \\
0 & \left(k_{\mathrm{L}}-k_{\mathrm{T}}\right) \sin \beta \cos \beta & k_{\mathrm{T}} \sin ^{2} \beta+k_{\mathrm{L}} \cos ^{2} \beta
\end{array}\right]
\end{aligned}
$$

where $\left[C^{\prime}\right]$ is a transformation matrix

$$
\left[C^{\prime}\right]=\left[\begin{array}{ccc}
1 & 0 & 0 \\
0 & \cos \beta & \sin \beta \\
0 & -\sin \beta & \cos \beta
\end{array}\right]
$$

Subsequently, the tensor $\mathbf{K}^{\prime \prime}$ in the cylindrical coordinate system $(R, \gamma, z)$ is transformed into the thermal conductivity tensor $\mathbf{K}$ in the Cartesian coordinate system $(x, y, z)$. This transformation is pretty easy:

$[\mathbf{K}]=\left[C^{\prime \prime}\right]\left[\mathbf{K}^{\prime \prime}\right]\left[C^{\prime \prime}\right]^{\mathrm{T}}=\left[\begin{array}{lll}k_{11} & k_{12} & k_{13} \\ k_{21} & k_{22} & k_{23} \\ k_{31} & k_{32} & k_{33}\end{array}\right]$

where

$\left[C^{\prime \prime}\right]=\left[\begin{array}{ccc}\cos \gamma & -\sin \gamma & 0 \\ \sin \gamma & \cos \gamma & 0 \\ 0 & 0 & 1\end{array}\right]$

The components of the tensor $\mathbf{K}$ for the helical tow are

$$
\begin{aligned}
& k_{11}=k_{\mathrm{T}} \cos ^{2} \gamma+\left(k_{\mathrm{T}} \cos ^{2} \beta+k_{\mathrm{L}} \sin ^{2} \beta\right) \sin ^{2} \gamma \\
& k_{12}=k_{21}=\left(k_{\mathrm{T}}-k_{\mathrm{L}}\right) \sin ^{2} \beta \sin \gamma \cos \gamma \\
& k_{13}=k_{31}=\left(k_{\mathrm{T}}-k_{\mathrm{L}}\right) \sin \beta \cos \beta \sin \gamma \\
& k_{22}=k_{\mathrm{T}} \sin ^{2} \gamma+\left(k_{\mathrm{T}} \cos ^{2} \beta+k_{\mathrm{L}} \sin ^{2} \beta\right) \cos ^{2} \gamma \\
& k_{23}=k_{32}=\left(k_{\mathrm{L}}-k_{\mathrm{T}}\right) \sin \beta \cos \beta \cos \gamma \\
& k_{33}=k_{\mathrm{T}} \sin ^{2} \beta+k_{\mathrm{L}} \cos ^{2} \beta
\end{aligned}
$$

The components of the thermal conductivity tensor $\mathrm{K}$ for the straight tow before the contact point are

$$
\begin{aligned}
& k_{11}=k_{\mathrm{T}} \\
& k_{12}=k_{21}=0 \\
& k_{13}=k_{31}=0
\end{aligned}
$$

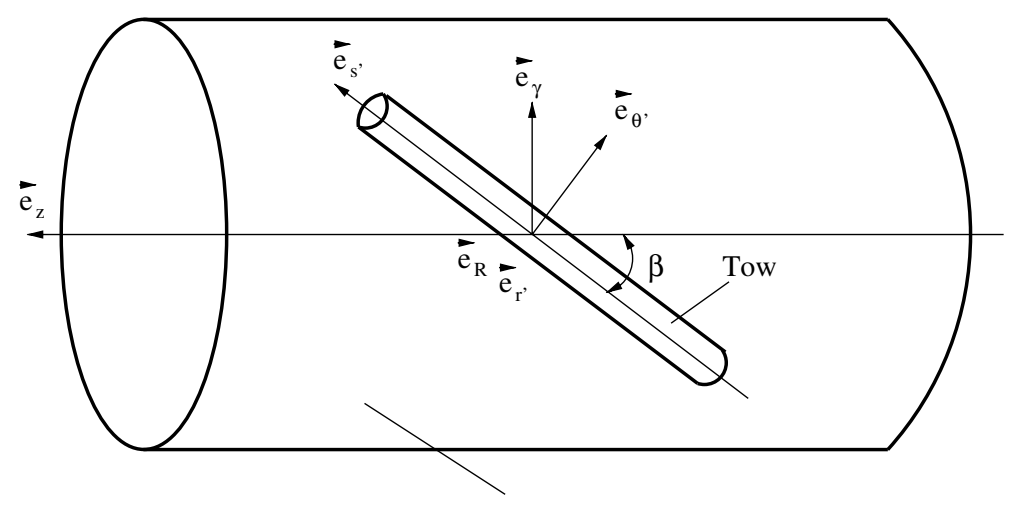

Fiber-wound composite structure

Fig. 3. Transformation of the thermal conductivity tensor of the tow in the cylindrical coordinates $\left(r^{\prime}, \theta^{\prime}, s^{\prime}\right)$ into that in the cylindrical coordinates $(R, \gamma, z)$ for the mandrel or fiber-wound composite structure. 
$k_{22}=k_{\mathrm{T}} \cos ^{2} \beta+k_{\mathrm{L}} \sin ^{2} \beta$

$k_{23}=k_{32}=\left(k_{\mathrm{L}}-k_{\mathrm{T}}\right) \sin \beta \cos \beta$

$k_{33}=k_{\mathrm{T}} \sin ^{2} \beta+k_{\mathrm{L}} \cos ^{2} \beta$

\subsection{Transformation of boundary conditions}

In the numerical grid generation method, transformation of boundary conditions from the physical domain to the computational domain is also required. As well known, the first kind of boundary condition will maintain the same after the transformation while the second kind of boundary condition is a special case of the third kind of boundary condition. Therefore, only the transformation of the third kind of boundary condition is needed. The third kind of boundary condition is given by

$\vec{n} \cdot \vec{q}=h\left(T-T_{\infty}\right)$

Here, the expression of unit vector normal to the surface of $\xi^{i}=$ constant is

$\vec{n}=\frac{\vec{a}^{i}}{\left|\vec{a}^{i}\right|}$

and the heat flux in an anisotropic medium, $\vec{q}$, is expressed by Eq. (29). The transformed third kind of boundary condition in the coordinate system $(\xi, \eta, \zeta)$ of the computational domain for $\xi^{i}=$ constant is

$-\frac{1}{\sqrt{g^{i i}}} \sum_{j=1}^{3} D_{i j} \frac{\partial T}{\partial \xi^{j}}=h\left(T-T_{\infty}\right) \quad(i=1,2,3)$

In the $(r, \theta, s)$ coordinate system, the transformed third kind of boundary conditions are: for $r=$ constant,

$$
\begin{aligned}
- & \left(B_{r 1} \cos \theta+B_{r 2} \sin \theta\right) \frac{\partial T}{\partial r}-\left(B_{\theta 1} \cos \theta+B_{\theta 2} \sin \theta\right) \frac{1}{r} \frac{\partial T}{\partial \theta} \\
- & \left(B_{s 1} \cos \theta+B_{s 2} \sin \theta\right) \frac{\partial T}{\partial s} \\
& =h\left(T-T_{\infty}\right)
\end{aligned}
$$

for $\theta=$ constant,

$$
\begin{aligned}
& \left(B_{r 1} \sin \theta-B_{r 2} \cos \theta\right) \frac{\partial T}{\partial r}+\left(B_{\theta 1} \sin \theta-B_{\theta 2} \cos \theta\right) \frac{1}{r} \frac{\partial T}{\partial \theta} \\
& \quad+\left(B_{s 1} \sin \theta-B_{s 2} \cos \theta\right) \frac{\partial T}{\partial s} \\
& \quad=h\left(T-T_{\infty}\right)
\end{aligned}
$$

for $s=$ constant,

$$
-B_{r 3} \frac{\partial T}{\partial r}-B_{\theta 3} \frac{1}{r} \frac{\partial T}{\partial \theta}-B_{s 3} \frac{\partial T}{\partial s}=h\left(T-T_{\infty}\right)
$$

where

$$
\begin{aligned}
& B_{r 1}=\frac{1}{\sqrt{g^{11}}}\left(D_{11} \cos \theta+D_{12} \sin \theta\right) \\
& B_{r 2}=\frac{1}{\sqrt{g^{22}}}\left(D_{21} \cos \theta+D_{22} \sin \theta\right) \\
& B_{r 3}=\frac{1}{\sqrt{g^{33}}}\left(D_{31} \cos \theta+D_{32} \sin \theta\right) \\
& B_{\theta 1}=\frac{1}{\sqrt{g^{11}}}\left(-D_{11} \sin \theta+D_{12} \cos \theta\right) \\
& B_{\theta 2}=\frac{1}{\sqrt{g^{22}}}\left(-D_{21} \sin \theta+D_{22} \cos \theta\right) \\
& B_{\theta 3}=\frac{1}{\sqrt{g^{33}}}\left(-D_{31} \sin \theta+D_{32} \cos \theta\right) \\
& B_{s 1}=\frac{1}{\sqrt{g^{11}}} D_{13}, \quad B_{s 2}=\frac{1}{\sqrt{g^{22}}} D_{23}, \\
& B_{s 3}=\frac{1}{\sqrt{g^{33}}} D_{33}
\end{aligned}
$$

\subsection{Grid generation results}

Fig. 4(a) and (b) exhibit the grids in the physical domain and the computational domain, respectively. For the tow of diameter $2 \mathrm{~mm}$ on the surface of a fiberwound composite structure of diameter $0.1 \mathrm{~m}$, the tow is relatively too small to be seen in details for grids. Therefore, here the grids for a tow of diameter $40 \mathrm{~mm}$ and $\beta=80^{\circ}$ on the surface of fiber-wound composite structure of diameter $0.1 \mathrm{~m}$ are generated and demonstrated. Only the grids on the surface of the tow are shown for clearness.

\section{Numerical method in computational domain}

Finite volume method [25] is applied to the numerical calculation in computational domain. Fully implicit time-marching and power-law schemes are employed in the discretization of the governing equation. To obtain the solution of the discretization equations, ADI iterative method is applied for the whole computational domain. For the solution of each point at each grid line, the standard TDMA is applied in the $r$ and $s$ directions. A CTDMA (Cyclic TDMA) [26,27] that is effective for periodic problems, in which the beginning and the end of the computational domain are the same, is applied in the $\theta$ direction. Because the source term and boundary condition are functions of temperature, they are calculated at each iteration step to obtain new values. The tolerance of iteration is $10^{-4}$. According to the cure kinetics model, strong nonlinearity occurs in the energy equation. Since the curing of resin is an integral process, 

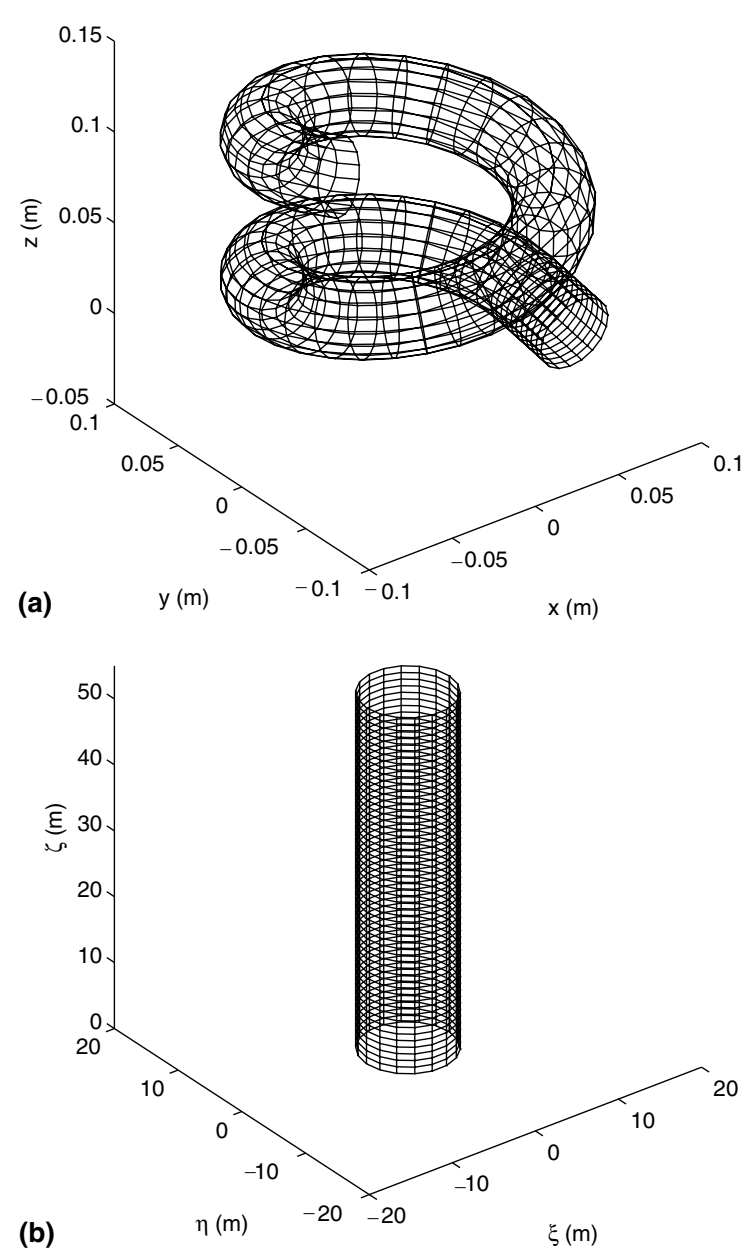

Fig. 4. Grids of the helical tow under the conditions of $D=0.1$ $\mathrm{m}, d=0.04 \mathrm{~m}$, and $\beta=80^{\circ}$ (only grids on the surface of the tow): (a) in the physical domain, (b) in the computational domain.

with the moving of the tow the increase of cure degree for each point of the tow within a small time step is calculated based on its cure degree at the previous time step.

\section{Results and discussion}

The on-line thermal curing process of thermoset composites in filament winding is investigated using the helical tow model and numerical grid generation method. A three-dimensional computer code is developed. At the beginning of numerical calculation, a comparison between the present numerical results and the existing data [3] is made. Chern et al. [3] studied in situ thermal curing of hoop-wound thermoset structure with infrared lamp. The prepreg tape with thickness of $0.132 \mathrm{~mm}$ is made of Hercules AS/3501-6. The surface of the structure is in convection with the ambient. Although the two models are different, a comparison of the results for the thermal curing processes can be made. The winding velocity $\left(V_{\mathrm{t}}=0.2 \mathrm{~m} / \mathrm{s}\right)$ and the diameter of the fiberwound composite structure $(D=0.2 \mathrm{~m})$ used in the present study for numerical result comparison are the same as those given in Ref. [3]. The comparison of the results are shown in Fig. 5. The temperature and cure degree profiles in the present study are at the centerline of the tow and those in Ref. [3] are at the middle of each layer of tape. It is seen that the present numerical results agree well with Chern's data.

The temperature and cure degree profiles under the conditions of $V_{\mathrm{t}}=0.1 \mathrm{~m} / \mathrm{s}, d=0.002 \mathrm{~m}, \beta=85^{\circ}, L_{1}=$ $10 d, L_{2}=10 d, L_{3}=175 d, T_{\mathrm{w}}=120^{\circ} \mathrm{C}, q=0.35 \times 10^{6}$ $\mathrm{W} / \mathrm{m}^{2}$, and different ratio of the diameter of fiber-wound composite structure to the diameter of tow are shown in Figs. 6 and 7, in which the results from the simplified
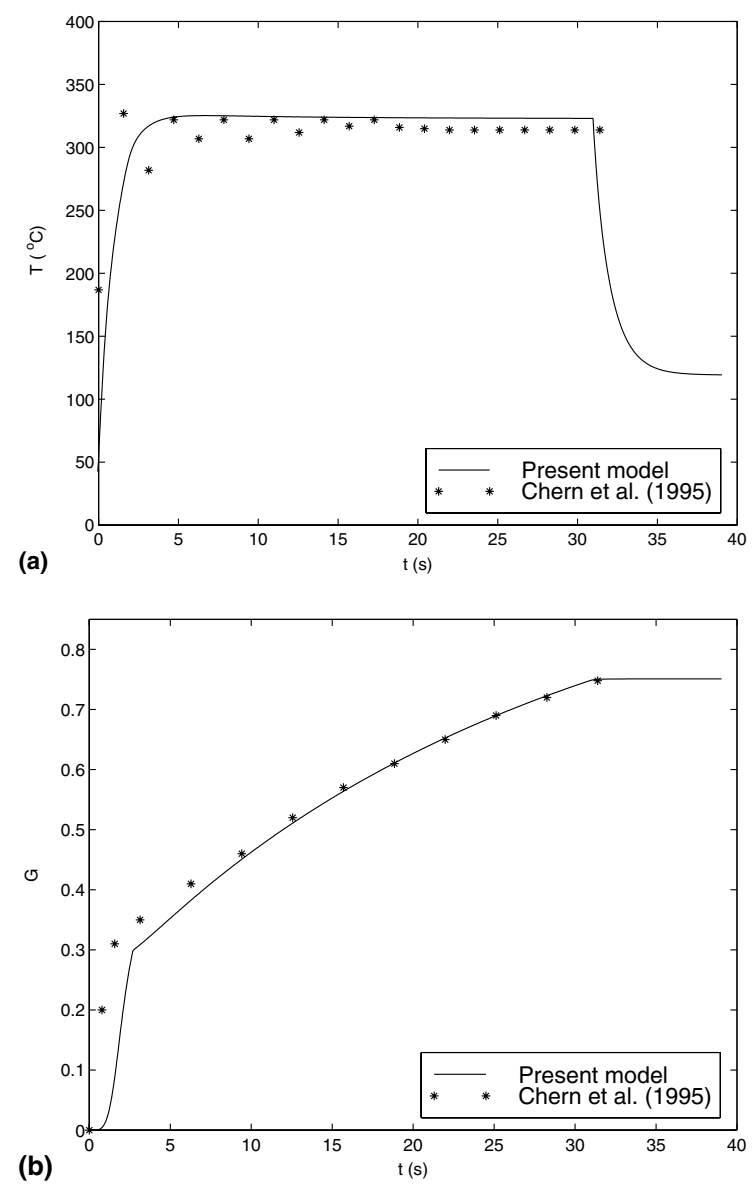

Fig. 5. Comparison of profiles of temperature and degree of cure: (a) temperature profiles, (b) cure degree profiles. 

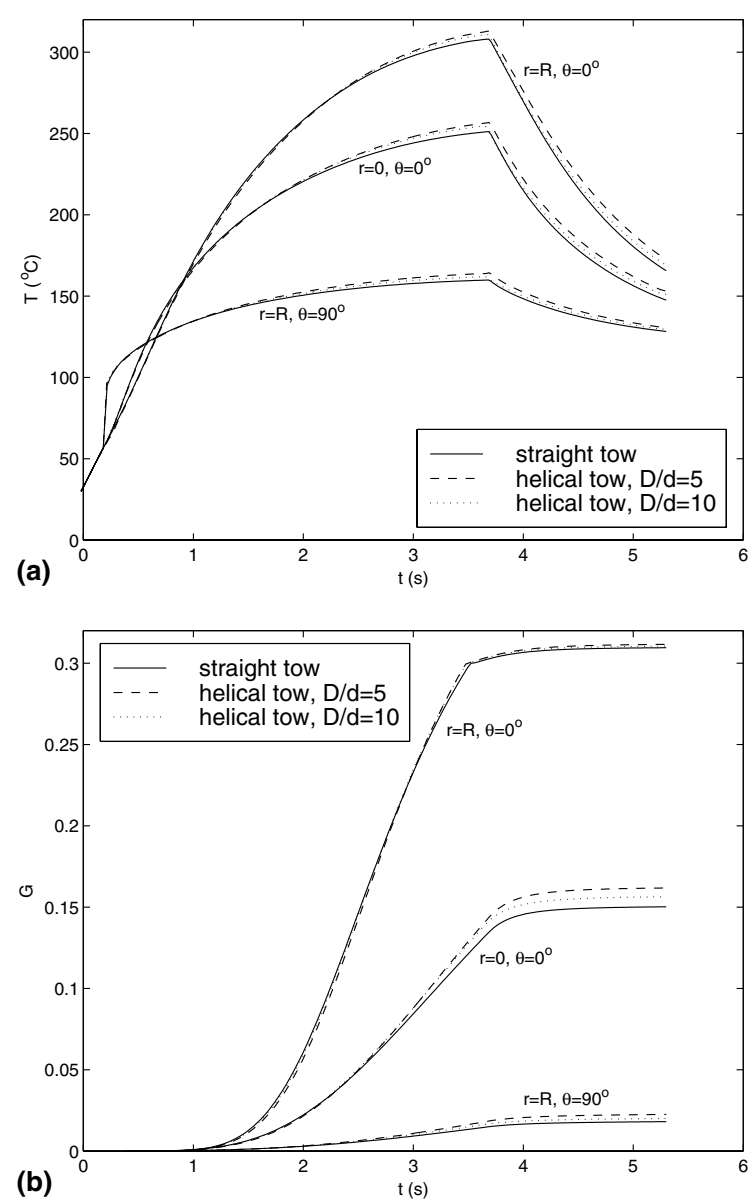

Fig. 6. Temperature and cure degree profiles at the centerline and several points on the surface of the tow under the conditions of $V_{\mathrm{t}}=0.1 \mathrm{~m} / \mathrm{s}, d=0.002 \mathrm{~m}, \beta=85^{\circ}, L_{1}=10 d, L_{2}=10 d$, $L_{3}=175 d, T_{\mathrm{w}}=120{ }^{\circ} \mathrm{C}$, and $q=0.35 \times 10^{6} \mathrm{~W} / \mathrm{m}^{2}$ : (a) temperature profiles, (b) cure degree profiles.

physical model (straight tow) $[4,5,9]$ are also shown for comparison. It is seen that the results obtained from both models coincide with each other very well when the ratio of the diameter of fiber-wound composite structure to the diameter of tow $(D / d)$ is larger than 100 . However, when the ratio is small, says 10 or 5 , the differences between the temperature and cure degree results obtained from both models become obvious and increase with time. Because the actual shape of the tow in filament winding process is considered in the helical tow model, results from this model are more accuracy than those from straight tow model. It is expected that the modeling of on-line thermal curing of thermoset composites in filament winding by the simplified physical model is only acceptable at large ratio. However, for the small ratio, the helical tow model should be used to obtain more accuracy results.
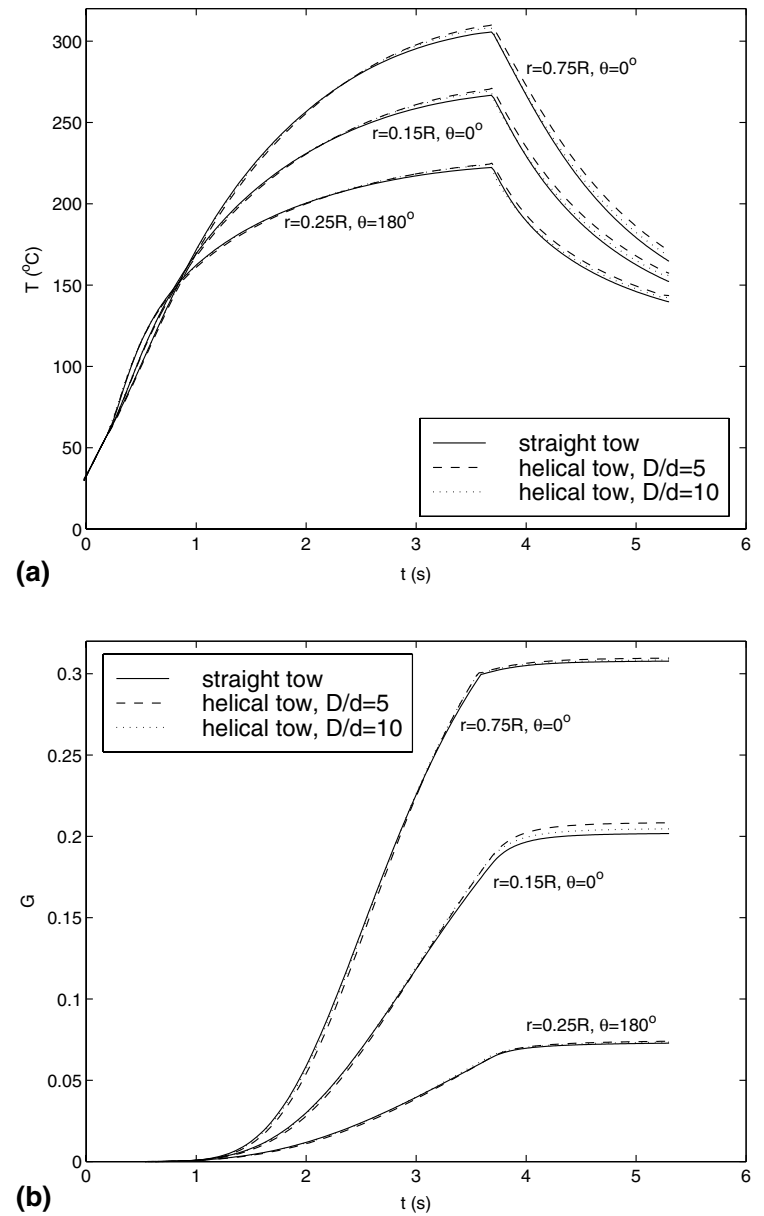

Fig. 7. Temperature and cure degree profiles at several points along the radial direction of the tow under the conditions of $V_{\mathrm{t}}=0.1 \mathrm{~m} / \mathrm{s}, \quad d=0.002 \mathrm{~m}, \quad \beta=85^{\circ}, \quad L_{1}=10 d, \quad L_{2}=10 d$, $L_{3}=175 d, T_{\mathrm{w}}=120{ }^{\circ} \mathrm{C}$, and $q=0.35 \times 10^{6} \mathrm{~W} / \mathrm{m}^{2}$ : (a) temperature profiles, (b) cure degree profiles.

\section{Conclusions}

A complicated physical model-helical tow model for the actual tow and the corresponding numerical method are presented, which successfully simulate the on-line thermal curing of thermoset composites in filament winding. A concise and regular transformed threedimensional energy equation and transformed boundary conditions for an anisotropic medium are derived, which are very suitable for numerical computation using the finite volume method. The results are compared to those obtained from the simplified physical model in which the curvature of the mandrel or the fiber-wound composite structure and tow is neglected. Obviously, the simplified physical model is acceptable only for the ratio of the diameter of fiber-wound composite structure to the 
diameter of tow is large. When the ratio is small, the helical tow model should be used.

\section{References}

[1] S.C. Mantell, G.S. Springer, Filament winding process models, Composite Structures 27 (1994) 141-147.

[2] V.N. Korotkov, Y.A. Chekanov, B.A. Rozenberg, The simultaneous process of filament winding and curing for polymer composites, Composites Science and Technology 47 (1993) 383-388.

[3] B.-C. Chern, T.J. Moon, J.R. Howell, Thermal analysis of in-situ curing for thermoset, hoop-wound structures using infrared heating: Part I-predictions assuming independent scattering, Transactions of the ASME, Journal of Heat Transfer 117 (1995) 674-680.

[4] X. Wang, Modeling of in-situ laser curing of thermosetmatrix composites in filament winding, Ph.D. thesis, University of Nebraska-Lincoln, Lincoln, NE, 2001.

[5] X. Wang, D.Y.S. Lou, Thermal modeling of in-situ laser curing of thermoset-matrix composites in filament winding, in: I. Dincer, M.F. Yardim (Eds.), Recent Advances in Transport Phenomena, Elsevier, 2000, pp. 659-664.

[6] X. Wang, D.Y.S. Lou, On-line laser curing of thermoset composites in filament winding, in: Proceedings of the First International Conference on Mechanical Engineering, Shanghai, China, November 20-22, 2000.

[7] X. Wang, D.Y.S. Lou, Numerical simulation of online laser curing of thermoset composites in filament winding, in: Proceedings of the 2nd ICHMT International Symposium on Advances in Computational Heat Transfer, Palm Cove, Queensland, Australia, May 20-25, 2001.

[8] X. Wang, D.Y.S. Lou, Improved model of online laser curing of thermoset composites in filament winding, in: Proceedings of the 4th International Thermal Energy Congress, Cesme, Izmir, Turkey, July 8-12, 2001, pp. 482488.

[9] X. Wang, D.Y.S. Lou, Simple thermal model for online laser curing of thermoset composites in filament winding, International Journal of Energy Research 27 (4) (2003) 377-388.

[10] C.Y. Wang, On the low-Reynolds-number flow in a helical pipe, Journal of Fluid Mechanics 108 (1981) 185-194.

[11] M. Germano, On the effect of torsion on a helical pipe flow, Journal of Fluid Mechanics 125 (1982) 1-8.

[12] M. Germano, The Dean equations extended to a helical pipe flow, Journal of Fluid Mechanics 203 (1989) 289-305.
[13] J.F. Thompson, F.C. Thames, C.W. Mastin, Automatic numerical generation of body-fitted curvilinear coordinate system for field containing any number of arbitrary twodimensional bodies, Journal of Computational Physics 15 (1974) 299-319.

[14] J.T. Tzeng, A.C. Loos, A cure analysis for axisymmetric composites, Composites Manufacturing 4 (3) (1993) 157165.

[15] W.I. Lee, A.C. Loos, G.S. Springer, Heat of reaction, degree of cure, and viscosity of Hercules 3501-6 resin, Journal of Composite Materials 16 (1982) 510-520.

[16] A.C. Loos, G.S. Springer, Curing of epoxy matrix composites, Journal of Composite Materials 17 (1983) 135-169.

[17] J.C. Seferis, C.N. Velisaris, Modeling-processing-structure relationships of polyetheretherketone (PEEK) based composites, in: 31st International SAMPE Symposium and Exhibition, April 7-10, 1986, pp. 1236-1252.

[18] G.S. Springer, S.W. Tsai, Thermal conductivities of unidirectional materials, Journal of Composite Materials 1 (1967) 166-173.

[19] C. Bachmann, UV light selection requires fitting lamp to application, Adhesive Age (1990) 19-21.

[20] N. Delahaye, J.M. Salter, M. Liziard, L. Podgorskl, Alkydbased thermosetting resins: influences of temperature and UV radiation on curing kinetics, Journal of Coatings Technology 67 (844) (1995) 67-70.

[21] G.A. Etemad, Free-convection heat transfer from a rotating horizontal cylinder to ambient air with interferometric study of flow, Transactions of the ASME 77 (8) (1955) 1283-1289.

[22] M.N. Ozisik, Finite Difference Methods in Heat Transfer, CRC Press, 1994.

[23] S. P. Kinsey, A numerical thermal model for the cure cycle of an advanced composite material, Ph.D. thesis, The University of Texas at Arlington, Arlington, 1991.

[24] W.J. Minkowycz, E.M. Sparrow, G.E. Schneider, R.H. Pletcher, Handbook of Numerical Heat Transfer, John Wiley \& Sons, 1988.

[25] S.V. Patankar, Numerical Heat Transfer and Fluid Flow, Hemisphere Publishing, Washington, DC, 1980.

[26] J.H. Ahlberg, E.N. Nilson, J.L. Walsh, The Theory of Splines and Their Applications, Academic Press, 1967.

[27] S.V. Patankar, C.H. Liu, E.M. Sparrow, Fully developed flow and heat transfer in ducts having streamwise-periodic variations of cross-sectional area, Transactions of the ASME, Journal of Heat Transfer 99 (2) (1977) 180186. 